\title{
Thermal conductivity of porous polymer materials considering pore special-shape and anisotropy
}

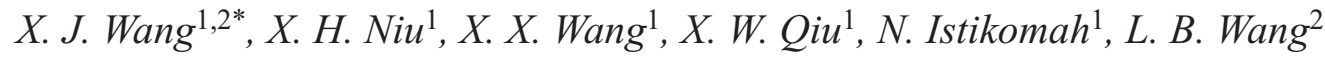 \\ ${ }^{1}$ School of Chemical and Biological Engineering, Lanzhou Jiaotong University, Lanzhou, 730070 Gansu, China \\ ${ }^{2}$ Key Laboratory of Railway Vehicle Thermal Engineering of Education Ministry, Lanzhou Jiaotong University, Lanzhou, \\ 730070 Gansu, China
}

Received 14 July 2020; accepted in revised form 10 September 2020

\begin{abstract}
The thermal insulation performance of porous polymer material is greatly affected by the pore structure. However, the effect of pore special-shape on the thermophysical properties of porous polymer material has not been considered. In this study, the effects of pore shape, overlap, and anisotropy are evaluated at the same time. The results show that the special-shaped pore had a better effect on the thermal insulation performance than common pores. The difference in thermal insulation performance caused by pore shape is increased with the gas content. The thermal insulation performance increases with the pore overlap only when the pores are overlapped in the direction vertical to heat flux. The common and specialshaped pores have different overlap probabilities in the same direction. The special-shaped pore is more sensitive to the directional angle than common pore and has significant anisotropy. Meanwhile, the pore anisotropy is affected by the pore overlap. The manufacture of porous polymer material must pay attention to the directional angle of the special-shaped pore and heat flux direction.
\end{abstract}

Keywords: polymer composites, there-dimensional model, anisotropy, special-shape

\section{Introduction}

Porous materials are widely used in agriculture, energy, metallurgy, environmental science, and chemical engineering [1-4]. In recent years, the use of porous materials increased in the construction industry that are required to have a thermal insulation performance for energy conservation [5]. The thermal conductivity is a basic factor in determining the heat preservation ability of porous materials. It has been considered that the thermal conductivity of porous material is not only dependent on thermal conductivities of components and gas content, but also on the pore geometries and microstructure of porous material [6]. So, understanding the effect of the aforementioned factors on the thermal conductivity is very important to design the porous material with excellent thermal insulation performance.
To obtain the influencing mechanisms of various characteristic parameters, the porous material must be prepared and then measured by test instruments. This has some disadvantages, such as complicated preparation process, more interference factors, high cost and time-consuming [7-9]. So, many theoretical models have been employed to predict the thermal conductivity of porous material and investigate the effect of pore geometries on it. The most commonly used models are the Series model, the Parallel model, the Maxwell-Eucken model, the Lewis-Nielsen model, and the Self-Consistent model [10-13]. These well-know models considered the pore with spherical, cubic, and ellipsoidal shapes and did not consider the effect of pore special-shape, overlap, and anisotropy. Many researchers have been proved that the shape of filler has a significant influence on 
the thermal conductivity of composite material. $\mathrm{Xu}$ et al. [14] investigated the effect of pore shape on the thermal conductivity of the porous composite. The pore shape includes sphere, icosahedron, dodecahedron, octahedron, cube, and tetrahedron. The results illuminated that the effect of pore shape closer to spherical shape is different from that for pore shape far from a spherical shape; the pore with special-shape may have some different rules. Babaei et al. [15] also investigated the effect of pore shape on the thermal conductivity of a series of idealized metal-organic porous material containing adsorbed gas using molecular simulations. They found that the thermal conductivity in porous material with triangular and hexagonal pore exhibits significant anisotropy. The porous material with hexagonal pore has both the highest and lowest thermal conductivities, parallel with and perpendicular to the heat flux direction, respectively. Several recent papers also discussed the effect of the pore shape and anisotropy on the porous materials in detail [16-19]. Moreover, some pieces of literature describe the relationship between the filler distribution and anisotropy could help to illustrate this relationship to be better [20,21].

Regretfully, the research results are missing for pore special-shape in previous literature reviews. The special-shaped components with I and $\mathrm{T}$ shapes have been widely used in heat and mass transfer. How do the special-shaped pores of $\mathrm{T}$ and I shaped (long edges, short edges, corners, and inflections) affect the thermal insulation performance of the porous polymer material remains unknown. Moreover, the combined effect of pore special-shape, overlap, and anisotropy has not been explicitly understudied. So, the aforementioned problems would be investigated at the same time in this paper. The heat transfer between pore gas and the matrix material was modeled by a three-dimensional heat conduction model. For comparison, the porous materials with sphere, cubic, and tetrahedral pore are also studied in the same way. Then they are used to reflect the effect of pore overlap, special-shape, and anisotropy on the thermal insulation performance of porous material. This study opens up fresh opportunities for developing super thermal insulation material.

\section{Numerical work}

\subsection{Generation of cell model}

Many three-dimensional models are used to study the thermal insulation performance of porous material.
The model has lots of pores, and can be calculated easily. The pore has two typical distributions and five shapes. The pore shape includes special-shapes (I shaped, T shaped) and common shapes (sphere, cubic, tetrahedral). The effect of pore overlap is researched by contrasting connected and isolated model. The pores are assigned randomly in a connected model; it has many interconnected pores. However, in a random model, the pores are distributed randomly in the matrix material without connected pore. The volume of a single pore is $0.002 \mathrm{~cm}^{3}$. All the pores with different shapes have the same capacity of the single pore. A $1 \times 1 \times 1 \mathrm{~cm}^{3}$ cube is selected for a cell model in this study. It has many pores and can represent a fractured surface of porous materials. The contact areas of single pore are $0.07677,0.09524$, $0.11439,0.14806$ and $0.16300 \mathrm{~cm}^{2}$ for sphere, cubic, tetrahedral, $\mathrm{T}$ shaped and I shaped. The contact areas of special-shaped pore are larger than common pore. In this research, the positions of pores are automatically generated by a computer program $[22,23]$. As shown in Figure 1, the positions of pore centers are represented by coordinates $\left(x_{0}, y_{0}, z_{0}\right)$, and the length, width, height of the model are denoted by $L_{\mathrm{x}}, L_{\mathrm{y}}, L_{\mathrm{z}}$, respectively. Then the random number $r_{\mathrm{x}}, r_{\mathrm{y}}$ and $r_{\mathrm{z}}$, are generated by the computer, which numbers line in $[0,1]$. The coordinate $x_{0}$ is selected as the product of $r_{\mathrm{x}}$ and $L_{\mathrm{x}}$. In a similar way, the coordinate $y_{0}$ and $z_{0}$ are obtained. In the process of pore generation for an isolated model, the overlaps of pores are canceled. When generating a connected model, the pore overlaps are retained. The pore generation process ends

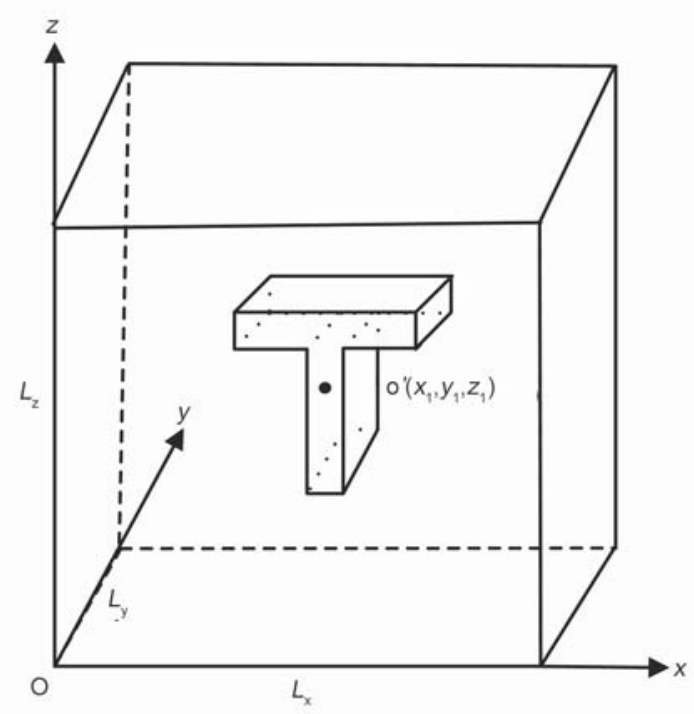

Figure 1. The distribution of a $\mathrm{T}$ shaped pore in three-dimensional model. 

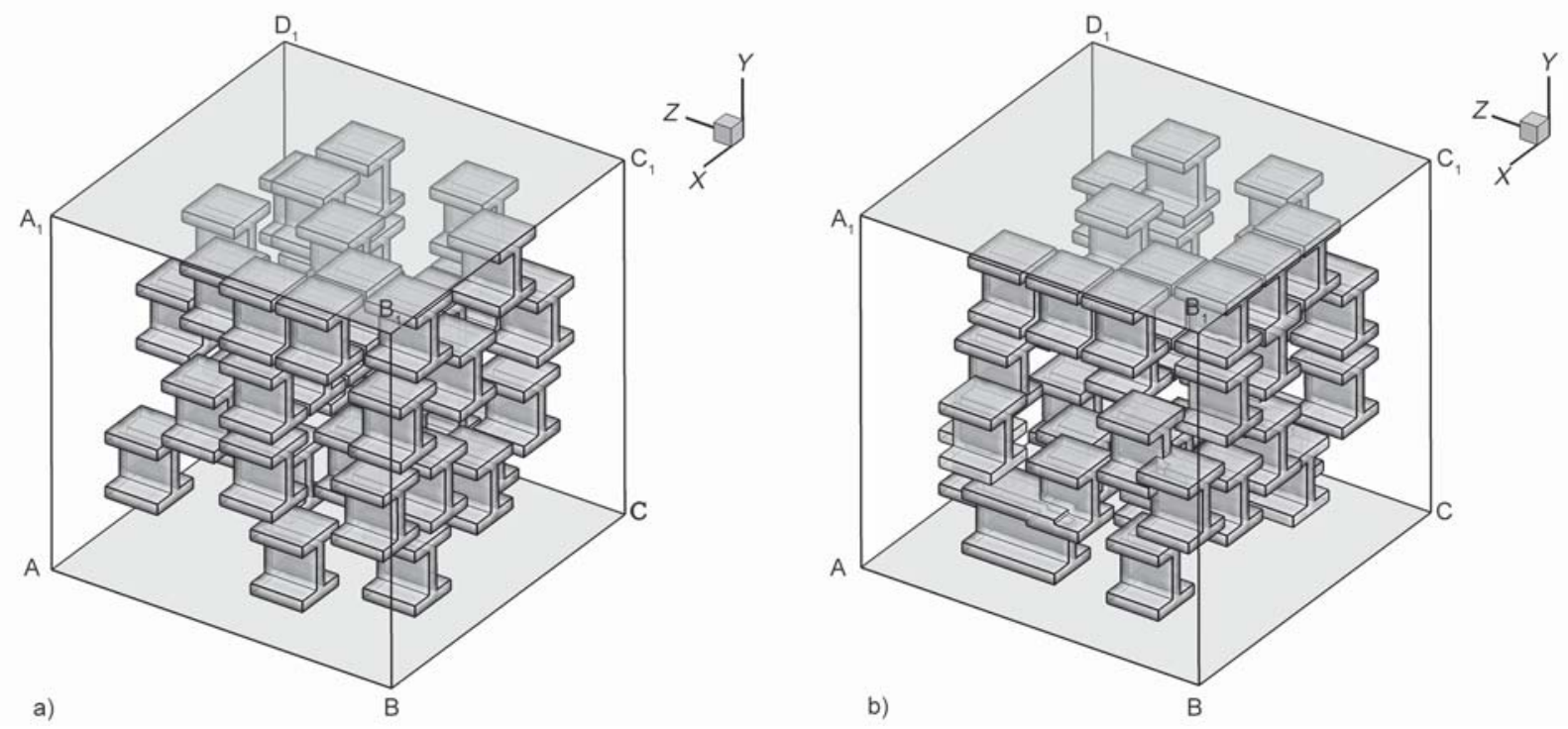

Figure 2. Three-dimensional numerical model comprising of I shaped pores and the matrix material, $V_{\mathrm{g}}=10 \%$ : (a) isolated model, (b) connected model.

when the desired pore content is reached. The isolated and connected models generated by the computer are shown in Figure 2. The pore shape is I shaped, and pore content is $10 \%$. As seen, the pore position in the isolated model is quite random and has no connected pores. But in the connected model, when a pore has overlap with one or more pores, a new larger pore is formed, and the thermal resistance path is extended. The number and size of such a large thermal resistance path increase with pore content. The directions of these paths are randomly in the matrix material. Meanwhile, the volume content of pore will be decreased with pore overlap. To reduce the randomness of pore overlap, ten models are generated for each pore contents. The effective thermal conductivity of these models is stable, and the difference

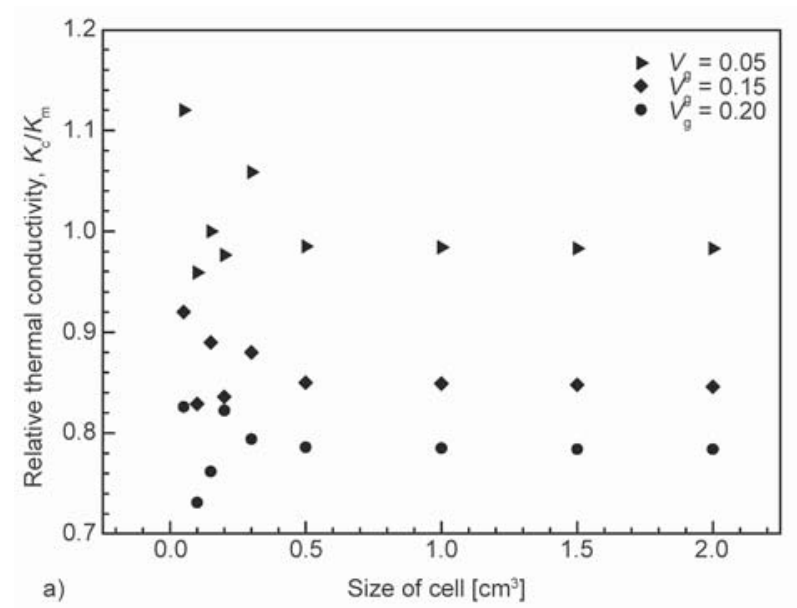

is within $3 \%$. The cell model has high stability and reliability.

The effects of model sizes on the relative thermal conductivity of porous material are shown in Figure 3. Relative thermal conductivity $\left(K_{\mathrm{c}} / K_{\mathrm{m}}\right)$ is the ratio of the thermal conductivity of porous material to the matrix material. The volume fractions of pore are 5 , 15 , and $20 \%$; the numerical models include isolated and connected models. As seen, it becomes stable, and the difference caused by randomness is no more than $2 \%$ when the cell model size is larger than $0.4 \mathrm{~cm}^{3}$. In this study, the cell size is $1 \times 1 \times 1 \mathrm{~cm}^{3}$. It is large enough for this numerical calculation. The volume content of pore is less than $20 \%$, and the design temperature of the porous polymer material is no more than $100^{\circ} \mathrm{C}$, meanwhile, the size of the pore

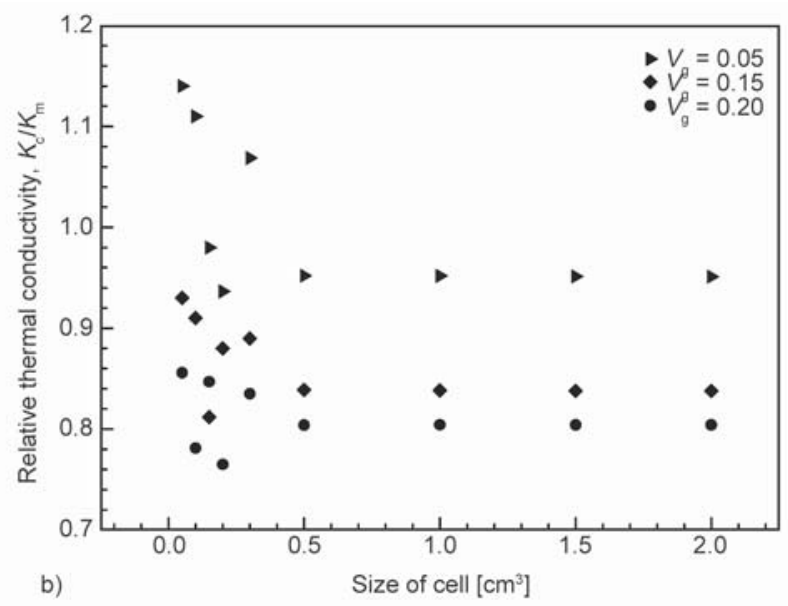

Figure 3. Effects of model size on the relative thermal conductivity of porous polymer material, I shaped pore: (a) isolated model, (b) connected model. 
is very small. So the effect of gas radiation and convection is very small and can be ignored. This study is a typical heat conduction problem.

\subsection{Heat conduction equations}

The heat conduction in the matrix material is satisfied by Equation (1) [24-25]:

$\frac{K_{\mathrm{m}}}{\left(\rho c_{\mathrm{p}}\right)_{\mathrm{m}}}\left(\frac{\partial^{2} T_{\mathrm{m}}}{\partial x^{2}}+\frac{\partial^{2} T_{\mathrm{m}}}{\partial y^{2}}+\frac{\partial^{2} T_{\mathrm{m}}}{\partial z^{2}}\right)=0$

The subscript ' $m$ ' denotes the matrix material, and the $x, y, z$ are the coordinates. In this numerical work, $K_{\mathrm{m}}$ is set to $0.104 \mathrm{~W} \cdot \mathrm{m}^{-1} \cdot \mathrm{K}^{-1}$, the same with silicone.

Heat conduction in the pore gas is given by Equation (2):

$\frac{K_{\mathrm{g}}}{\left(\rho c_{\mathrm{p}}\right)_{\mathrm{g}}}\left(\frac{\partial^{2} T_{\mathrm{g}}}{\partial x^{2}}+\frac{\partial^{2} T_{\mathrm{g}}}{\partial y^{2}}+\frac{\partial^{2} T_{\mathrm{g}}}{\partial z^{2}}\right)=0$

The subscript ' $g$ ' denotes pore gas. $K_{\mathrm{g}}$ is set to $0.023 \mathrm{~W} \cdot \mathrm{m}^{-1} \cdot \mathrm{K}^{-1}$, the same with air.

The second class boundary condition (see Equation (3)) is set on the surface of $A_{1} B_{1} C_{1} D_{1}$ (top flux), $\mathrm{A}_{1} \mathrm{ABB}_{1}$ (front flux), or $\mathrm{A}_{1} \mathrm{ADD}$ ) (side flux):

$-\left.K_{\mathrm{m}} \frac{\partial T}{\partial n}\right|_{\Gamma}=q$

The $q$ is the area mean total heat flux. In this study, $q$ is set to $1000 \mathrm{~W} / \mathrm{m}^{2}$.

A third class boundary condition is set on the bottom surface of the cell, $\mathrm{ABCD}$ (top flux), $\mathrm{D}_{1} \mathrm{DCC}_{1}$ (front flux) or $\mathrm{B}_{1} \mathrm{BCC}_{1}$ (side flux) is given by Equation (4):

$-\left.K_{\mathrm{m}} \frac{\partial T}{\partial n}\right|_{\Gamma}=\left.h\left(T-T_{\mathrm{f}}\right)\right|_{\Gamma}$

The convective heat transfer coefficient $(h)$ is set to $20 \mathrm{~W} \cdot \mathrm{m}^{-1} \cdot \mathrm{K}^{-1}$. The ambient temperature $\left(T_{\mathrm{f}}\right)$ is set to $300 \mathrm{~K}$.

The other four boundary conditions are given by Equation (5):

$\left.\frac{\partial T}{\partial n}\right|_{\Gamma}=0$

They are assumed adiabatic. The input parameters don't affect the thermal conductivities inside the numerical models. These are used for the convenience of the numerical calculation.

\subsection{Heat conduction coupling}

The isothermal boundary condition is set on the interface between the matrix material and pore gas is given by Equation (6):

$T_{\mathrm{m}, \text { interface }}=T_{\mathrm{g}, \text { interface }}$

When the temperature fields in the cell are calculated, the effective thermal conductivity in the $y$-direction is estimated by Equation (7):

$K_{\mathrm{c}}=\frac{q L_{\mathrm{y}}}{T_{\mathrm{t}}-T_{\mathrm{b}}}$

where $q$ is the area mean heat flux at a cross-section normal to the $y$-direction. $T_{\mathrm{t}}$ and $T_{\mathrm{b}}$ are the constant temperatures obtained by integrating the temperature at the top and bottom surface.

\subsection{Numerical simulation and model validation}

In order to validate the accuracy of the numerical simulation, the effective thermal conductivities of porous material with spherical pore are calculated to compare with some well-known models [10-13]. The expressions of these well-known models are listed as Equation (8)-(12):

Series model:

$K_{\mathrm{c}}=\frac{K_{\mathrm{g}} K_{\mathrm{m}}}{K_{\mathrm{g}}\left(1-V_{\mathrm{g}}\right)+K_{\mathrm{m}} V_{\mathrm{g}}}$

Parallel model:

$K_{\mathrm{c}}=K_{\mathrm{g}} V_{\mathrm{g}}+K_{\mathrm{m}}\left(1-V_{\mathrm{g}}\right)$

Maxwell-Eucken model:

$K_{\mathrm{c}}=\frac{2 K_{\mathrm{m}}+K_{\mathrm{g}}-2\left(K_{\mathrm{m}}-K_{\mathrm{g}}\right) V_{\mathrm{g}}}{2 K_{\mathrm{m}}+K_{\mathrm{g}}+\left(K_{\mathrm{m}}-K_{\mathrm{g}}\right) V_{\mathrm{g}}}$

Lewis-Nielsen model:

$K_{\mathrm{c}}=K_{\mathrm{m}}\left[\frac{1+A B V_{\mathrm{f}}}{1-B V_{\mathrm{f}} \Psi}\right], \quad B=\frac{\frac{K_{\mathrm{g}}}{K_{\mathrm{m}}}-1}{\frac{K_{\mathrm{g}}}{K_{\mathrm{m}}}+A}$,

$\Psi=1+\left(\frac{1-V_{\mathrm{r}}}{V_{\mathrm{r}}^{2}}\right) V_{\mathrm{g}}, \quad A=k_{\mathrm{E}}-1$

Self-Consistent model:

$$
K_{\mathrm{c}}=\frac{1}{4}\left[\left(3 V_{\mathrm{g}}-1\right) K_{\mathrm{g}}+K_{\mathrm{m}}\left(3\left(1-V_{\mathrm{g}}\right)-1\right)+\sqrt{\left[\left(3 V_{\mathrm{g}}-1\right) K_{\mathrm{g}}+\left(3\left(1-V_{\mathrm{g}}\right)-1\right)\right]^{2}+8 K_{\mathrm{g}} K_{\mathrm{m}}}\right]
$$




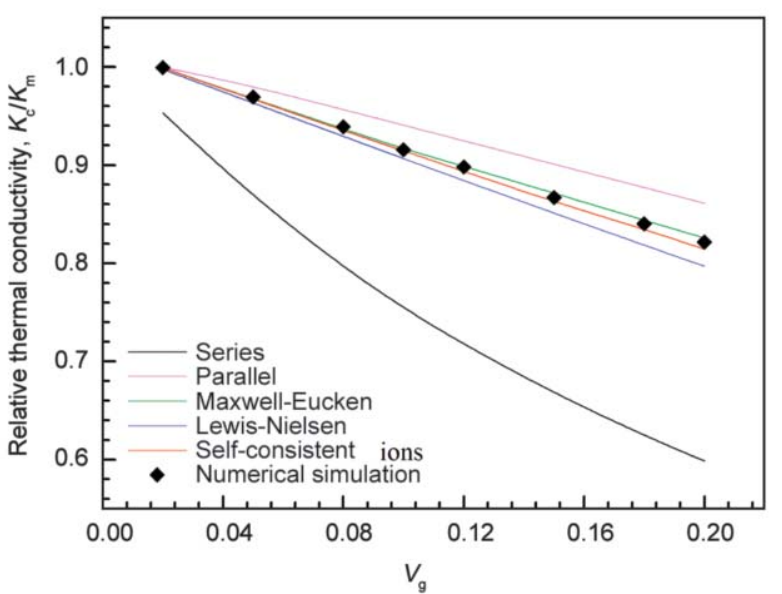

Figure 4. Comparison of numerical simulation with other well-know models.

The thermal conductivities of the matrix material and the pore gas are 0.104 and $0.023 \mathrm{~W} \cdot \mathrm{m}^{-1} \cdot \mathrm{K}^{-1}$. Lewis-Nielsen model with $A=1.5$ and $V_{\mathrm{r}}=0.637$ are used in this study [13]. The results of these models and numerical simulations are shown in Figure 4. It indicates that the numerical results are highly consistent with Maxwell-Eucken and Self-Consistent model under the whole range of pore gas content from 0 to $20 \%$; the maximum deviation is less than $0.6 \%$. The result deviation is also no more than $3 \%$ under the whole range between the numerical simulation and Lewis-Nielsen model. The numerical analysis method has high reliability.

To assure the accuracy of the results calculated, a grid independence test is conducted. The pore shape is I shaped, and the volume content of pore gas is $10 \%$. These pores are distributed randomly in the matrix material, as shown in Figure 2a. The relative thermal conductivities with various grid densities are shown in Table 1. Meanwhile, the time consumptions of gridding and calculation are also showed in Table 1. As seen, the relative thermal conductivity tend to a definite value when the grid density more than $50 \times 50 \times 50$. The difference of the relative thermal conductivity is no more than $0.35 \%$ caused by $50 \times 50 \times 50$ grids and $100 \times 100 \times 100$ grids. An $50 \times 50 \times 50$ grid is used for this numerical calculation.

Table 1. Time consumed in calculation with different mesh numbers for an isolated model with 10\% I pores.

\begin{tabular}{|l|c|c|c|}
\hline Mesh number & $\begin{array}{c}\text { Meshing time } \\
{[\mathbf{s}]}\end{array}$ & $\begin{array}{c}\text { Calculation } \\
\text { time } \\
{[\text { min] }}\end{array}$ & $\begin{array}{c}\text { Relative } \\
\text { thermal } \\
\text { conductivity }\end{array}$ \\
\hline $25 \times 25 \times 25$ & 310 & 240 & 0.8905 \\
\hline $50 \times 50 \times 50$ & 960 & 770 & 0.8898 \\
\hline $100 \times 100 \times 100$ & 7450 & 6200 & 0.8895 \\
\hline
\end{tabular}

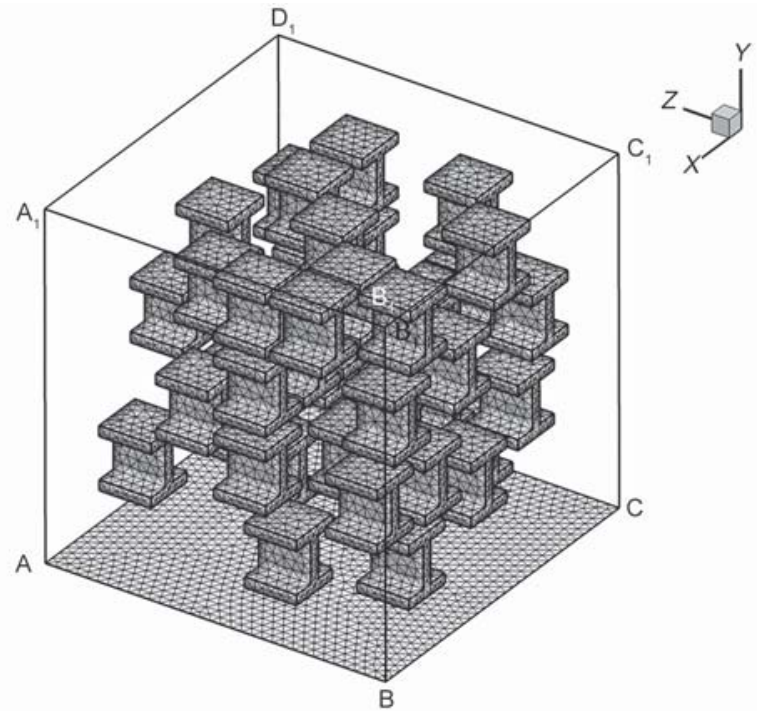

Figure 5. The structure of computational grids (only showing the bottom surface and pores).

It can save significant times. The absolute convergence criterion in the energy equation is up to $10^{-12}$ for all models. The computational mesh of the cell and pores are shown in Figure 5. To see clearly the pores inside the cell, only the meshes on the bottom surface of the cell are plotted.

\section{Results and discussion}

\subsection{Effect of pore shape}

The effect of pore special-shape on the heat conduction of porous materials is revealed by isolated and connected model separately. The relative thermal conductivities of the porous material with isolated pore are shown in Figure 6. The matrix material is silicone with a thermal conductivity of $0.104 \mathrm{~W} \cdot \mathrm{m}^{-1} \cdot \mathrm{K}^{-1}$. The gas in the pore is air with a

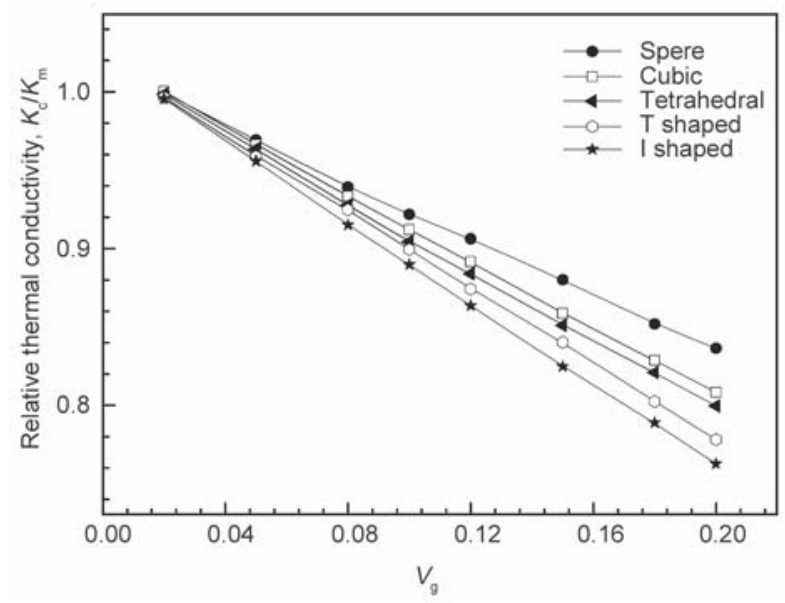

Figure 6. Relative thermal conductivity of porous polymer material with different shaped pores: isolated model. 

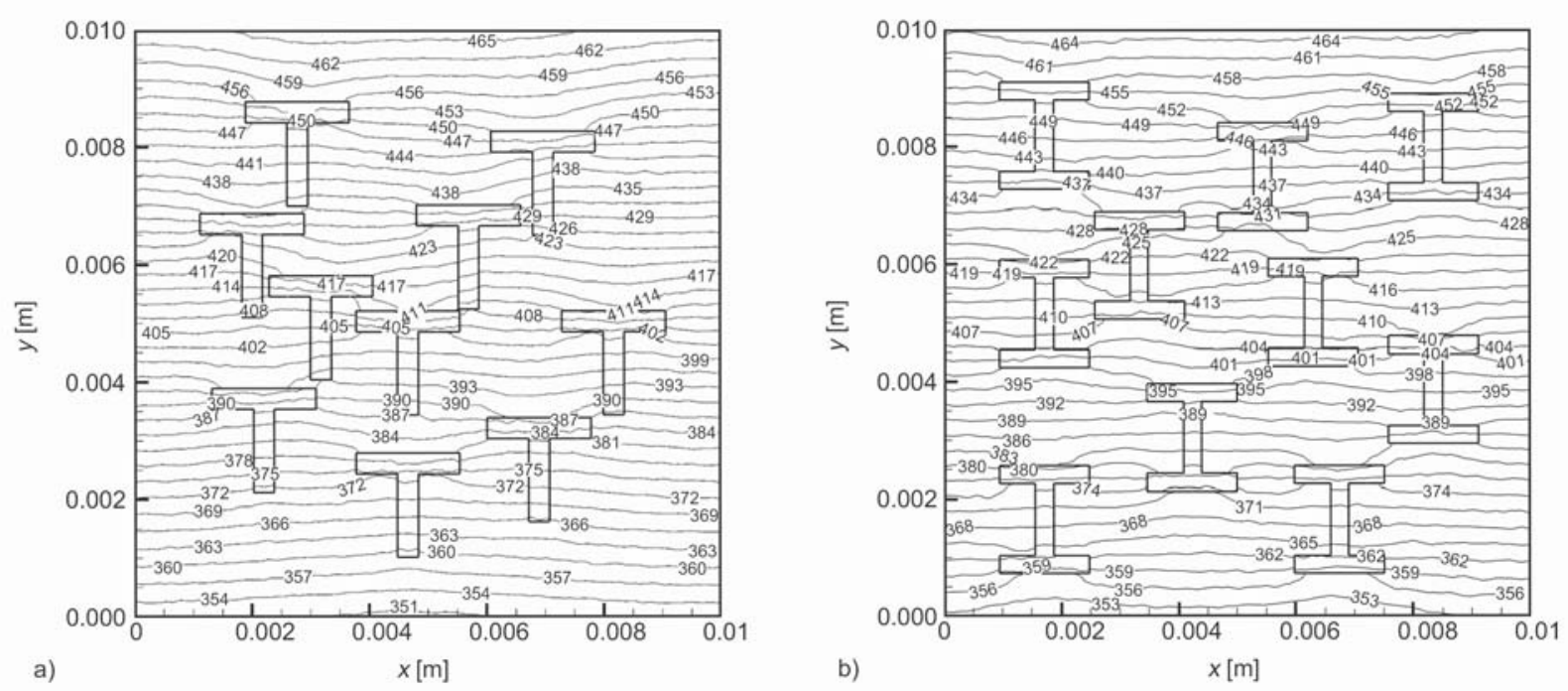

Figure 7. Isotherms $[\mathrm{K}]$ on the centered $x-y$ plane $\left(z=0.5 L_{\mathrm{z}}\right)$ in isolated model, $V_{\mathrm{g}}=0.20$ : (a) T shaped pore, (b) I shaped pore.

thermal conductivity of $0.023 \mathrm{~W} \cdot \mathrm{m}^{-1} \cdot \mathrm{K}^{-1}$. The gas content increases from 2 to $20 \%$. As seen, the relative thermal conductivities of porous material are always linearly decreased with gas content for common and special-shaped pore. But, the pore with different shapes has different decrease speeds. The decrease rate of the special-shaped pore is larger than the common pore. The order for thermal conductivity decreasing is: I shaped $>$ T shaped $>$ tetrahedral $>$ cubic $>$ sphere. The differences caused by pore shape become more and more apparent with the increase of gas content. At the same gas content of 5 and $10 \%$, the difference of the thermal conductivity is 1.4 and $3.4 \%$ between I shaped and sphere pore, respectively. By contrast, the difference is more than $9.7 \%$ at a gas content of $20 \%$. The difference is due to the pore shape. The special-shaped pore has a substantial effect on the thermal insulation performance of porous material in an isolated model.

The effect of pore gas can be explained by the isotherms in the unit cell. Figure 7 shows the isotherms with a special-shaped pore. The pore shapes are $\mathrm{T}$, and I shaped, and the pore content of $20 \%$. The thermal resistance of air is far greater than matrix material, so the isotherms in a matrix material are deformed and closed by air. The pore gas act obstacles, delaying the heat fluxes through them. The heat fluxes flow around the obstacle in the matrix material. The effective contact area is the main factor affecting the heat transfer, across the heat flux direction. The transfer rate decreases obviously across the contact area. The length of the pore has a smaller effect on the heat transfer along the heat flux direction. They play a secondary role in the obstacle of heat transfer. The special-shaped pore has a larger effective contact area at upstream and downstream and has a longer thermal resistance path along the heat flux direction. So, the special-shaped pore has the optimal thermal insulation in an isolated model. The common-shaped pores (tetrahedral, cubic, sphere) have a short heat resistance path. Meanwhile, the effective contact area is also small in the direction vertical to heat flux. The thermal isolation performance of a common-shaped pore is lower than a special-shaped pore.

Figure 8 shows the relative thermal conductivities of porous material with connected pores. The order of thermal conductivity decreasing is the same as in the isolated model. However, the change rule of thermal conductivity is different from the isolated model. At the same gas contents of 5,10 , and $20 \%$, the difference

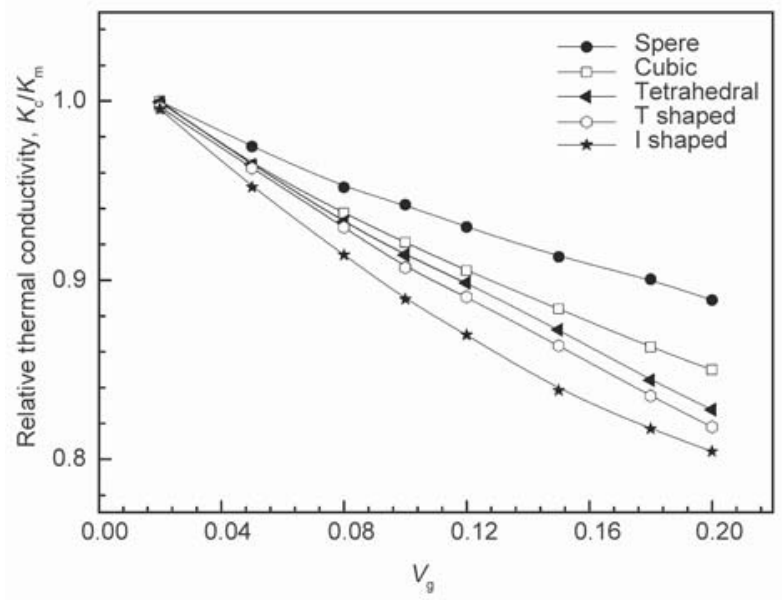

Figure 8. Relative thermal conductivity of porous polymer material with different shaped pores: connected model. 
of thermal conductivity between I shaped and sphere pore is $2.3,5.6$, and $10.5 \%$, respectively. It is larger than in the isolated model. This is caused by pore overlap. Some large-pores are formed by the pore overlap. The size of these large-pores increases with pore content. When the pores are overlapped in the direction vertical to heat flux, the effective contact areas are increased with the pore overlap. When they are overlapped along the heat flux direction, the effective contact areas (vertical to heat flux) are decreased. They have different overlap probabilities in two directions for a common and a special-shaped pore.

Meanwhile, the total volume content of pore gas would be reduced by the pore overlap, and the trends become more and more significant with the pore number. The actual volume content changes of different shaped pores are shown in Figure 9. As seen, the volume content of spherical pore has the largest

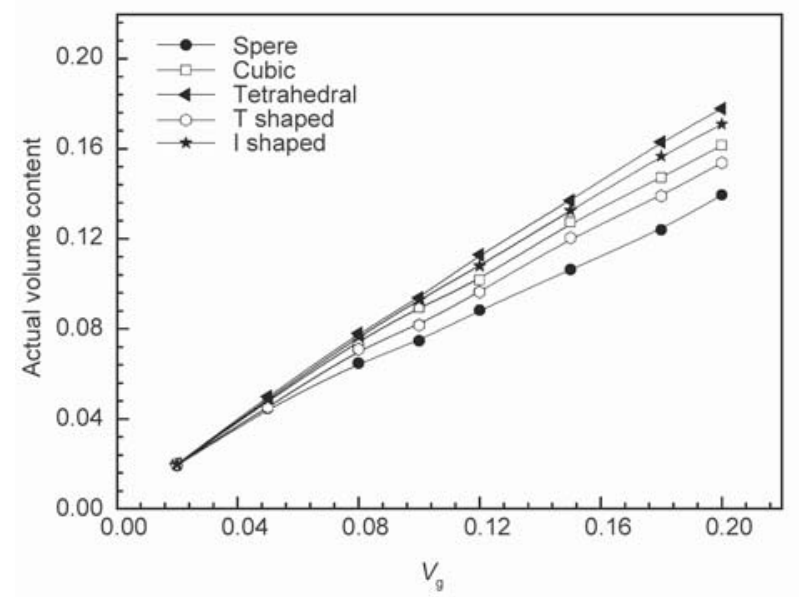

Figure 9. The change of volume content for connected model.

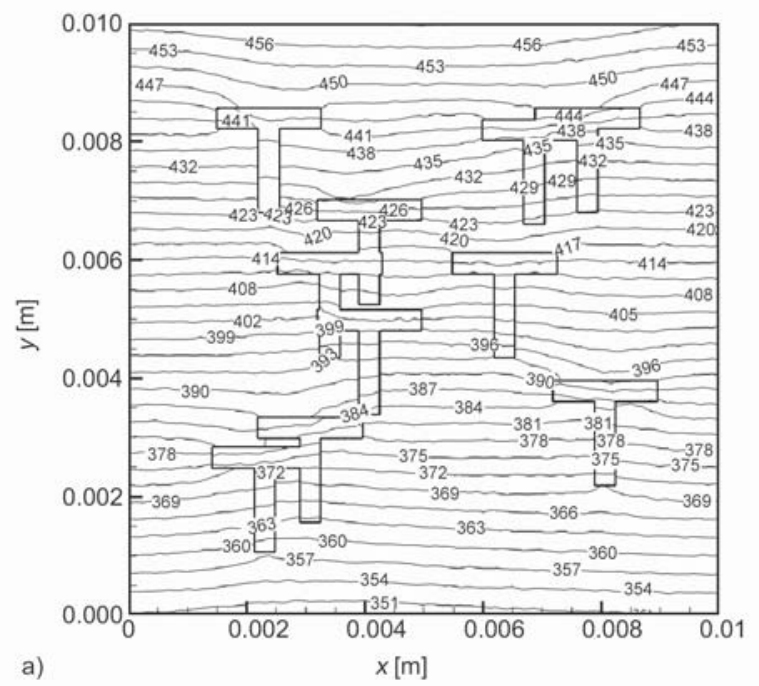

decrease with the pore number, followed by $\mathrm{T}$ shaped pore, and the tetrahedral pore is the least. For example, at the theoretical volume content of $20 \%$, the actual volume content is reduced by $30 \%$ for sphere pore. At the same volume content, it is $11 \%$ for tetrahedral pore. The thermal conductivity of porous material with pore overlap is affected by large-pore and volume decrease. The thermal insulation performance of a special-shaped pore ( $\mathrm{T}$ shaped and I shaped) is also larger than a common pore in the connected model.

Figure 10 shows the effect of pore gas on the isotherms in the connected model. Many large-pores are formed by pore overlap. The large-pores maybe distribute in the direction vertical to heat flux or along the heat flux direction. The deformed and closed degrees of isotherms around these large-pores (vertical to heat flux) are very serious. However, when the large-pores along the heat flux direction, the deformed and closed degrees of isotherms are very little and similar to straight lines. Pore overlap has a significant effect on the thermal insulation performance of porous material when they are distributed in the direction vertical to the heat flux. New methods to control the overlap direction of pore should be investigated for increasing the chance of pore overlap in the direction vertical to heat flux.

\subsection{Effect of pore anisotropy}

The special-shapes (T and I shaped) are different from the sphere. They are more sensitive to the directional angle. They have significant anisotropy. The pore distribution and heat flux direction often determine

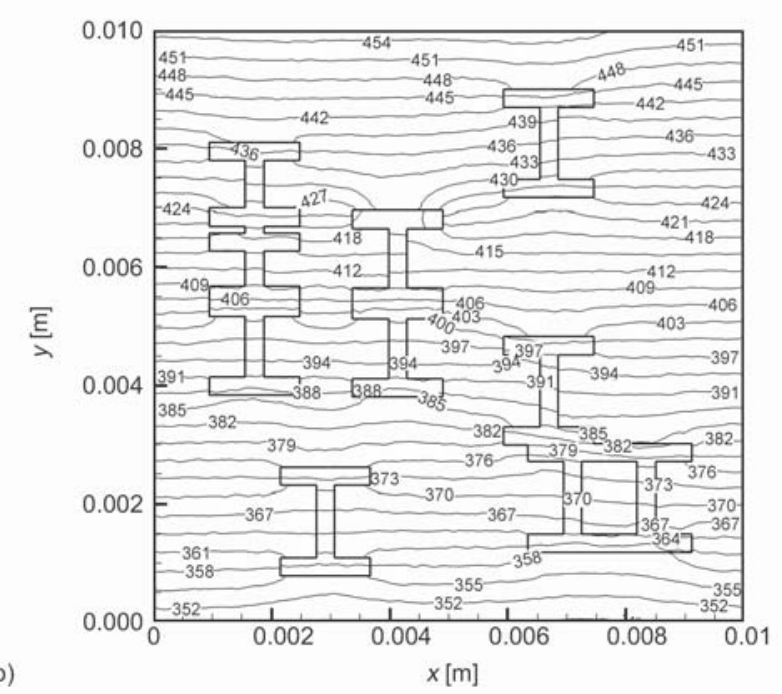

Figure 10. Isotherms $[\mathrm{K}]$ on the centered $x-y$ plane $\left(z=0.5 L_{\mathrm{z}}\right)$ in connected model, $V_{\mathrm{g}}=0.20$ : (a) T shaped pore, (b) I shaped pore. 
the thermal insulation performance of porous material. In order to reveal the anisotropy of I and T shaped pore, the heat flux would across the numerical model from three directions. In the first case, the heat flux flows into the top surface $\left(\mathrm{A}_{1} \mathrm{~B}_{1} \mathrm{C}_{1} \mathrm{D}_{1}\right)$ and out from the bottom surface $(A B C D)$; in the second case, it flows into the front surface $\left(\mathrm{A}_{1} \mathrm{ABB}_{1}\right)$ and out from the back surface $\left(\mathrm{D}_{1} \mathrm{DCC}_{1}\right)$; in the third case, it flows into the left surface $\left(\mathrm{A}_{1} \mathrm{ADD}_{1}\right)$ and out from the right surface $\left(\mathrm{B}_{1} \mathrm{BCC}_{1}\right)$. The anisotropies of specialshaped pore are researched by isolated and connected model, respectively.

Figure 11 shows the relative thermal conductivities of porous material with different heat flux directions. The results are calculated by isolated models. For porous material with I shaped pore, the thermal insulation performance with top heat flux is much larger than the front and side heat flux. At the same gas content of $20 \%$, the thermal conductivity of top heat flux is lower $11.5 \%$ than side heat flux. The thermal conductivity has little difference between front heat flux and side heat flux. When the porous material with top heat flux, I shaped pore has two large effective contact areas at the upstream and downstream. It can impede the heat flux twice. I shaped pore has the best thermal insulation performance with top heat flux. For porous material with $\mathrm{T}$ shaped pore, the order of thermal insulation performance with different heat flux directions is front flux $>$ top flux $>$ side flux. The difference is very small between top flux and front flux. However, the performance of side heat flux is far lower than other both cases. At the same gas content of $20 \%$, the difference of thermal conductivity is $0.5 \%$ between top heat flux and front heat flux. The difference is $9.3 \%$ between side

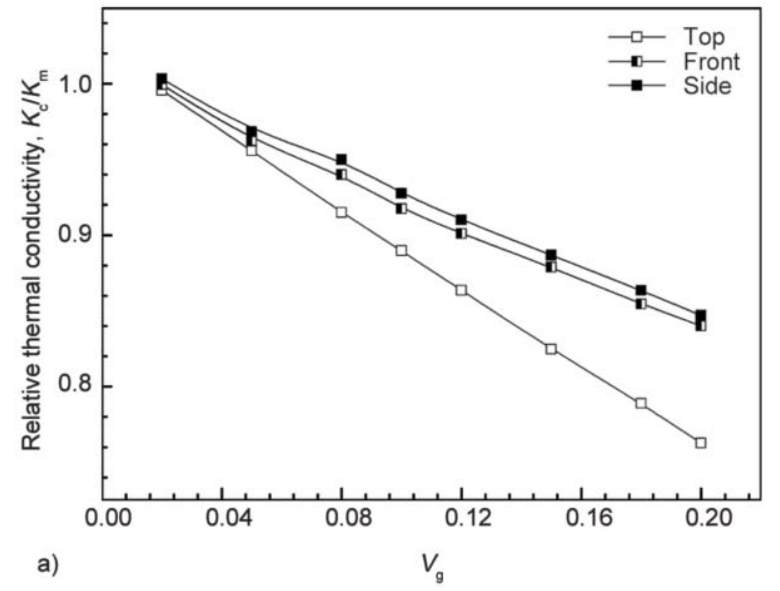

heat flux and top heat flux. T shaped pore with top heat flux and front heat flux has the same contact areas in the direction vertical to heat flux, but it is small for porous material with side heat flux. So, the thermal insulation performance of porous material with top and front heat flux is larger than side heat flux.

The sphere is a perfectly symmetrical shape. The contact area (vertical to heat flux) is not affected by the heat flux direction. The cubic and tetrahedral are similar to the symmetrical structure. They have the same contact area in three heat flux directions. The thermal conductivities of porous material have a small difference in different heat flux directions for sphere, cubic, and tetrahedral pores.

Figure 12 shows the relative thermal conductivities of porous material from connected models. As seen, the rules are consistent with the isolated model. However, the difference of thermal conductivity is larger than the isolated model. At the same gas content of $20 \%$, the difference is $0.8 \%$ between front and side heat flux for I shaped pore in isolated model, and it increases to $4 \%$ in the connected model. This is because the difference of contact area is increased by the pore overlap. I shaped pore with top heat flux also has the largest thermal insulation performance, and it is the front heat flux for $\mathrm{T}$ shaped pore.

\section{Conclusions}

In this study, the finite volume method is used to calculate the thermal conductivity of porous polymer material. The effects of pore special-shape, overlap, and anisotropy are researched at the same time. From the numerical results, the following results can be concluded:

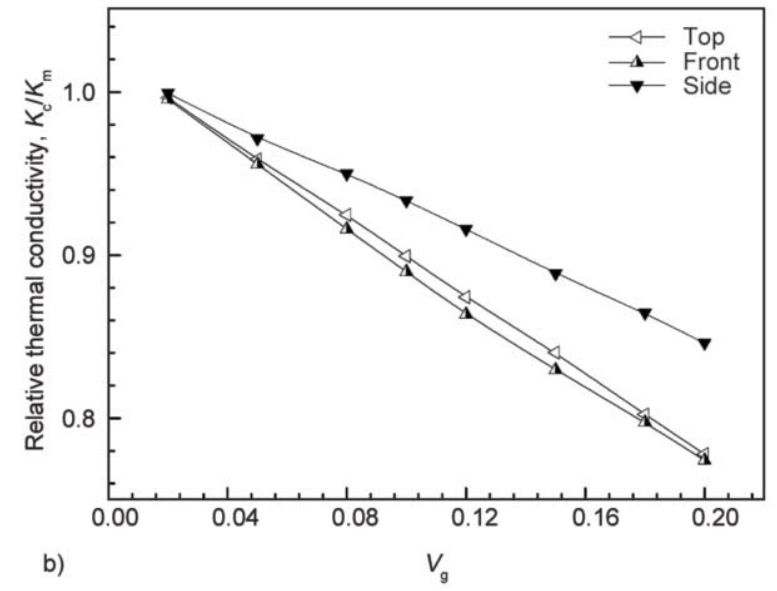

Figure 11. Anisotropic thermal conductivity of porous polymer material in isolated model: (a) I shaped pore, (b) T shaped pore. 

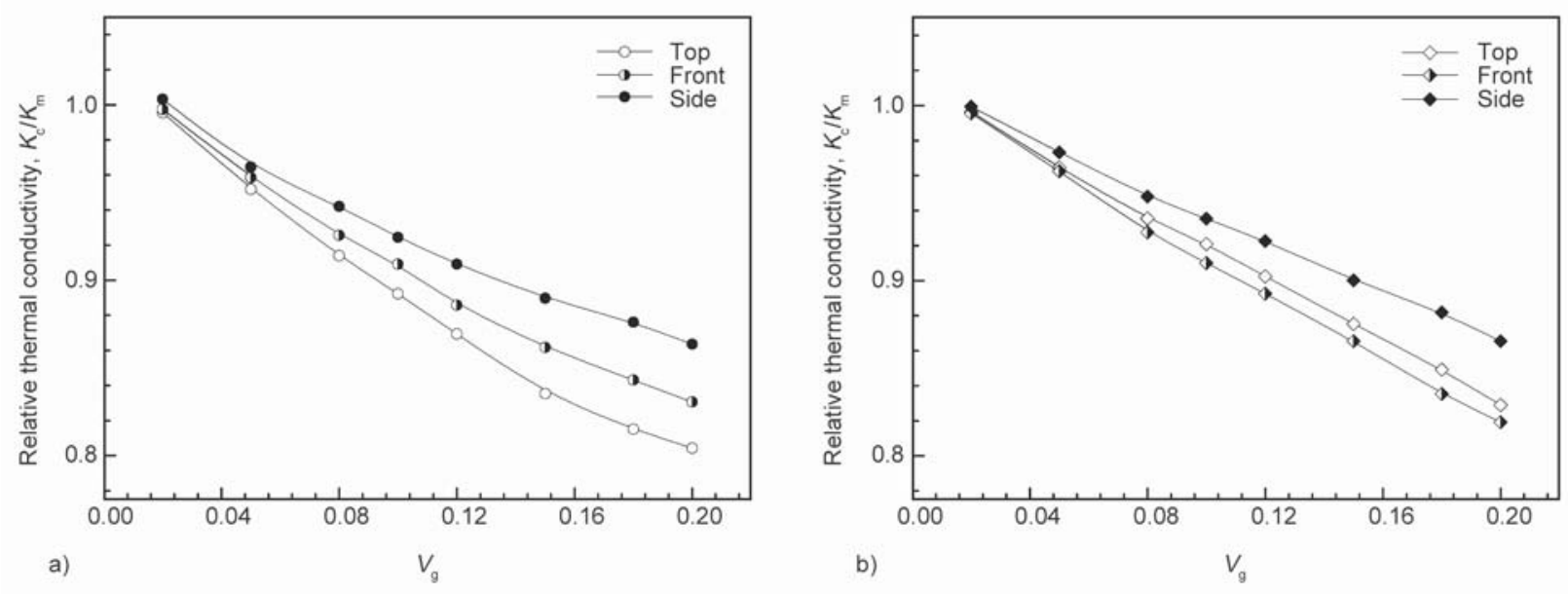

Figure 12. Anisotropic thermal conductivity of porous polymer material in connected model: (a) I shaped pore, (b) T shaped pore.

(1) The relative thermal conductivities of porous material are always linearly decreased with gas content, regardless of the pore shapes. But, the pore with different shapes has different decrease speeds. The decrease rate of the special-shaped pore is larger than common pore. The differences caused by pore shape become more and more apparent with gas content.

(2) When the pores are overlapped in the direction vertical to heat flux, the thermal insulation performance of porous material is increased with the pore overlap. When they are overlapped along the heat flux direction, the performance is decreased. The overlap probabilities are different in two directions for common and specialshaped pore.

(3) The thermal insulation performance of specialshaped pores is more sensitive to directional angle than common pores and has significant anisotropy. For porous material with I shaped pore, the thermal insulation performance with top heat flux is much larger than the front and side heat flux. For T shaped pore, the order of thermal insulation performance with different heat flux directions is front flux $>$ top flux $>$ side flux. Meanwhile, the pore anisotropies are affected by the pore overlap.

\section{Acknowledgements}

This Project is supported by the Foundation of A Hundred Youth Talents Training Program of Lanzhou Jiaotong University. It is also supported by the National Natural Science Foundation of China, No.51406072.

\section{Nomenclatures}

$h$

$h \quad$ convective heat transfer coefficient $\left[\mathrm{W} \cdot \mathrm{m}^{-2} \cdot \mathrm{K}^{-1}\right]$

$K \quad$ thermal conductivity $\left[\mathrm{W} \cdot \mathrm{m}^{-2} \cdot \mathrm{K}^{-1}\right.$ ]

$L_{\mathrm{x}} \quad$ length along the $x$ axis

$L_{\mathrm{y}} \quad$ width along the $y$ axis

$L_{\mathrm{z}} \quad$ height along the $z$ axis

$n \quad$ normal direction

$N \quad$ number of pores

$q \quad$ heat flux $\left[\mathrm{W} \cdot \mathrm{m}^{-2}\right]$

$T \quad$ temperature $[\mathrm{K}]$

$T_{\mathrm{f}} \quad$ ambient temperature [K]

$V \quad$ volumetric fraction

$x, y, z \quad$ coordinates [m]

$x_{0} \quad$ coordinate of pore center $[\mathrm{m}]$

$y_{0} \quad$ coordinate of pore center $[\mathrm{m}]$

$z_{0} \quad$ coordinate of pore center [m]

Subscripts

porous material

pore gas

polymer matrix

\section{References}

[1] Xie Z., Duan F. L.: Material processing for low thermal conductivity for advanced aero-engine applications. Results in Materials, 6, 100079/1-100079/7 (2020).

https://doi.org/10.1016/j.rinma.2020.100079

[2] Mujeebu M. A., Abdullah M. Z., Abu Bakar M. Z., Mohamad A. A., Abdullah M. K.: Applications of porous media combustion technology - A review. Applied Energy, 86, 1365-1375 (2009).

https://doi.org/10.1016/j.apenergy.2009.01.017

[3] Gong L., Wang Y., Chen X., Zhang R., Zhang H.: Thermal conductivity of highly porous mullite materials. International Journal of Heat and Mass Transfer, 67, 253259 (2013).

https://doi.org/10.1016/j.ijheatmasstransfer.2013.08.008 
[4] Delgado J. M. P. Q.: Heat and mass transfer in porous media. Springer, Berlin (2012). https://doi.org/10.1007/978-3-642-21966-5

[5] Qiu L., Zou H., Tang D., Wen D., Feng Y., Zhang X.: Inhomogeneity in pore size appreciably lowering thermal conductivity for porous thermal insulators. Applied Thermal Engineering, 130, 1004-1011 (2018). https://doi.org/10.1016/j.applthermaleng.2017.11.066

[6] Hussain M., Tao W-Q.: Thermal conductivity of composite building materials: A pore scale modeling approach. International Journal of Heat and Mass Transfer, 148, 118691/1-18691/11 (2020). https://doi.org/10.1016/j.ijheatmasstransfer.2019.118691

[7] Zhang M., He M., Gu H., Huang A., Xiang W.: Influence of pore distribution on the equivalent thermal conductivity of low porosity ceramic closed-cell foams. Ceramics International, 44, 19319-19329 (2018). https://doi.org/10.1016/j.ceramint.2018.07.160

[8] Han Y., Lv S., Hao C., Ding F., Zhang Y.: Thermal conductivity enhancement of BN/silicone composites cured under electric field: Stacking of shape, thermal conductivity, and particle packing structure anisotropies. Thermochimica Acta, 529, 68-73 (2012). https://doi.org/10.1016/j.tca.2011.11.029

[9] Chen Z., Cheng P., Hsu C. T.: A theoretical and experimental study on stagnant thermal conductivity of bidispersed porous media. International Communications in Heat and Mass Transfer, 27, 601-610 (2000). https://doi.org/10.1016/S0735-1933(00)00142-1

[10] El Moumen A., Kanit T., Imad A., El Minor H.: Computational thermal conductivity in porous materials using homogenization techniques: Numerical and statistical approaches. Computational Materials Science, 97, 148-158 (2015).

https://doi.org/10.1016/j.commatsci.2014.09.043

[11] Dai Z., Nawaz K., Park Y. G., Bock J., Jacobi A. M.: Correcting and extending the Boomsma-Poulikakos effective thermal conductivity model for three-dimensional, fluid-saturated metal foams. International Communications in Heat and Mass Transfer, 37, 575-580 (2010). https://doi.org/10.1016/j.icheatmasstransfer.2010.01.015

[12] Maxwell J. C.: A treatise on electricity and magnetism. Nature, 7, 478-480 (1873). https://doi.org/10.1038/007478a0

[13] Lewis T. B., Nielsen L. E.: Dynamic mechanical properties of particulate-filled composites. Journal of Applied Polymer Science, 14, 1449-1471 (1970). https://doi.org/10.1002/app.1970.070140604

[14] Xu W., Jia M., Gong Z.: Thermal conductivity and tortuosity of porous composites considering percolation of porous network: From spherical to polyhedral pores. Composites Science and Technology, 167, 134-140 (2018). https://doi.org/10.1016/j.compscitech.2018.07.038

[15] Babaei H., McGaughey A. J. H., Wilmer C. E.: Effect of pore size and shape on the thermal conductivity of metal-organic frameworks. Chemical Science, 8, 583589 (2017).

https://doi.org/10.1039/C6SC03704F
[16] Li H., Zeng Q., Xu S.: Effect of pore shape on the thermal conductivity of partially saturated cement-based porous composites. Cement and Concrete Composites, 81, 87-96 (2017). https://doi.org/10.1016/j.cemconcomp.2017.05.002

[17] Qiu L., Li Y. M., Zheng X. H., Zhu J., Tang D. W., Wu J. Q., Xu. C. H.: Thermal-conductivity studies of macroporous polymer-derived SiOC ceramics. International Journal of Thermophysics, 35, 76-89 (2014). https://doi.org/10.1007/s10765-013-1542-8

[18] Yang L., Du K., Zhang X.: A theoretical investigation of thermal conductivity of nanofluids with particles in cylindrical shape by anisotropy analysis. Powder Technology, 314, 328-338 (2017).

https://doi.org/10.1016/j.powtec.2016.09.032

[19] Leclerc W., Ferguen N., Pélegris C., Bellenger E., Guessasma M., Haddad H.: An efficient numerical model for investigating the effects of anisotropy on the effective thermal conductivity of alumina/Al composites. Advances in Engineering Software, 77, 1-12 (2014). https://doi.org/10.1016/j.advengsoft.2014.07.004

[20] Wang X., Wang X., Liu M., Crimp M., Wang Y., Qu Z.: Anisotropic thermal expansion coefficient of multilayer graphene reinforced copper matrix composites. Journal of Alloys and Compounds, 755, 114-122 (2018). https://doi.org/10.1016/j.jallcom.2018.04.325

[21] Wang W. L., Li B., Qu Z. G., Zhang J. F., Jin Z. G.: Effects of graphite microstructure evolution on the anisotropic thermal conductivity of expanded graphite/paraffin phase change materials and their thermal energy storage performance. International Journal of Heat and Mass Transfer, 155, 119853/1-119853/9 (2020). https://doi.org/10.1016/j.ijheatmasstransfer.2020.119853

[22] Wang X., Liu H., Wang L., Liu B., Wang L.: Optimization of thermal insulation performance for the porous materials. Heat Transfer Engineering, 39, 1573-1581 (2018). https://doi.org/10.1080/01457632.2017.1369847

[23] Wang X. J.: The effect of the prismatic filler arrangement andcross-sectional shape on the thermal conductivity of polymercomposites. Express Polymer Letters, 12, 920-931 (2014). https://doi.org/10.3144/expresspolymlett.2014.93

[24] Rao Z., Chen B., Zhao J.: A series of generalized correlations for predicting the thermal conductivity of composite materials packing with artificially designed filler shapes. Applied Thermal Engineering, 120, 444452 (2017). https://doi.org/10.1016/j.applthermaleng.2017.04.002

[25] Wang X., Liu H., Qiu X., Wang L., Wang L.: Thermal conductivity of filled composite materials considering interactions between fillers. Applied Thermal Engineering, 141, 835-843 (2018). https://doi.org/10.1016/j.applthermaleng.2018.06.022 